Journal of Engineering and Applied Sciences 14 (7): 2171-2176, 2019

ISSN: 1816-949X

(C) Medwell Journals, 2019

\title{
Local Quadrant Pattern with Co-occurrence Matrix (LQP-CM): Hybrid Method for Image Classification and Feature Extraction
}

\author{
Hassan Mohammed Mahdi Al-Jawahry and Hind Rustum Mohammed \\ Department of Computer Science, Faculty of Computer Science and Mathematics, \\ University of Kufa, Kufa, Iraq
}

\begin{abstract}
Image classification is important in several fields which depend on the methods of extracting the features. This study proposes a new method for features extraction called Local Quadrant Pattern with Co-occurrence Matrix (LQP-CM) that related with Local Ternary Pattern (LTP) and Gray-Level Co-occurrence Matrix (GLCM). LQP-CM will map the values into four types instead of two like Local Binary Pattern (LBP) or three like LTP. For classification, this study will use the Euclidean Distance (ED) to classifying the features that extracting. The data set that used in this study is Brodatz dataset. The MATLAB environment was adopted in the programming and the criteria was used to evaluate the performance of the proposed method is percentage of correct classification which proved successful in classification the database used in high efficiency.
\end{abstract}

Key words: Local quadrant pattern, local quadrant pattern with co-occurrence matrix, local binary pattern, local ternary pattern, gray-level co-occurrence matrix, classification, Euclidean distance

\section{INTRODUCTION}

Image classification is an important and interesting subject for many researchers. The classification of images is used in many areas for example in the field of medical classification of the disease. It also entered in classification of texture images. There were many methods in this area which depend on the extraction of texture information, like Gray-Level Co-occurrence Matrix (GLCM) (Haralick et al., 1973; Haralick, 1979), Texture Spectrum (TS) (He and Wang, 1991), LBP (Heikkila and Pietaikinen, 2006) and LTP (Tan and Triggs, 2007). The researchers have developed these methods to get better results for features extraction and classification. The features extraction is depending on the local texture properties for each pixel of images and thus get many of statistics.

This study proposes new method for extract features and classification called Local Quadrant Pattern with Co-occurrence Matrix (LQP-CM) which it is new version of local patterns. LQP-CM is depending on LTP with GLCM but in LQP-CM use quadrant encoding instead of ternary or binary. In LQP-CM use eight neighbors of pixel for extract texture information, then encoding values to one of four types, then produce the upper and lower matrix.

The dataset that used in this paper is Brodatz dataset and Euclidean distance used for classification by calculating the distance between features of testing image and features of each training images and get the minimum distance.

\section{Literature review}

Gray-level co-occurrence matrix: GLCM is statistical method to extract texture features from matrix produced by calculating transitions between pairs of two pixels. This matrix with size equal to maximum value of origin matrix or image. GLCM method is introduced by Haralick et al. (1973) and Haralick (1979) where he used it for image classification by extract feature from GLCM. It's depended on two main parameters, one is $d$ called distance and the other is angular $\theta$. Where $\mathrm{d}$ is distance between two pixels and è is the direction must go and it may be equal to 045 90135 . GLCM can be defined as next Eq. 1:

$$
p(i, j / d, \theta)=\frac{N_{d, \theta}(i, j)}{N}
$$

where, $\mathrm{N}$ is summation of all transitions. Next is example to explain how GLCM is research: Let matrix in Fig. 1a is origin matrix, $d=1$ and $\theta=0$. So we will create matrix with size $\max (a) \times \max (a)$ as matrix in Fig. 1c. the value of first location in matrix $\mathrm{b}(1,1)$ calculating by counting how many pixels with value 1 in origin matrix have neighbor with value 1 in left and right with distance 1 equal to $\mathrm{d}$. Also, location $(1,2)$ in matrix $b$ is the counting of pixels with value 1 and its neighbor have value 2 in left and right. And so on for all locations and for all angular.

Corresponding Author: Hassan Mohammed Mahdi Al-Jawahry Department of Computer Science, Faculty of Computer Science and Mathematics, University of Kufa, Kufa, Iraq 
(a)

$$
\begin{array}{|l|l|l|l|}
\hline 1 & 1 & 0 & 2 \\
\hline 0 & 0 & 3 & 3 \\
\hline 2 & 2 & 1 & 1 \\
\hline 2 & 1 & 0 & 1 \\
\hline
\end{array}
$$

(c)

\begin{tabular}{|l|l|l|l|l|}
\hline & 0 & 1 & 2 & 3 \\
\hline 0 & 2 & 3 & 1 & 1 \\
\hline 1 & 3 & 4 & 2 & 0 \\
\hline 2 & 1 & 2 & 2 & 0 \\
\hline 3 & 1 & 0 & 0 & 2 \\
\hline
\end{tabular}

(e)

\begin{tabular}{|l|l|l|l|l|}
\hline & 0 & 1 & 2 & 3 \\
\hline 0 & 0 & 3 & 2 & 1 \\
\hline 1 & 3 & 2 & 1 & 2 \\
\hline 2 & 2 & 1 & 2 & 1 \\
\hline 3 & 1 & 2 & 1 & 0 \\
\hline
\end{tabular}

(b)

\begin{tabular}{|c|c|c|c|c|}
\hline & 0 & 1 & 2 & 3 \\
\hline 0 & 2 & 2 & 1 & 0 \\
\hline 1 & 2 & 2 & 0 & 1 \\
\hline 2 & 1 & 0 & 2 & 2 \\
\hline 3 & 0 & 1 & 2 & 0 \\
\hline \multicolumn{5}{|l|}{ (f) } \\
\hline 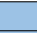 & 0 & 1 & 2 & 3 \\
\hline 0 & 0 & 2 & 2 & 1 \\
\hline 1 & 2 & 2 & 1 & 2 \\
\hline 2 & 2 & 1 & 0 & 0 \\
\hline 3 & 1 & 2 & 0 & 0 \\
\hline
\end{tabular}

\begin{tabular}{c|c|c|c|c|}
\multicolumn{1}{c}{$\mathbf{0}$} & \multicolumn{1}{c}{$\mathbf{2}$} & $\mathbf{2}$ & $\mathbf{3}$ \\
\hline 0 & 0,0 & 0,1 & 0,2 & 0,3 \\
\cline { 2 - 5 } 1 & 1,0 & 1,1 & 1,2 & 1,3 \\
\cline { 2 - 5 } 2 & 2,0 & 2,1 & 2,2 & 2,3 \\
\cline { 2 - 5 } 3 & 3,0 & 3,1 & 3,2 & 3,3 \\
\cline { 2 - 5 } & & & &
\end{tabular}

(d)
Fig. 1: Framework of GLCM; a) Origin matrix; b) Mains value with neighbour value in origin matrix; $c$ ) Matrix with $\theta=0$; d) Matrix with $\theta=45$; e) Matrix with $\theta=90$;) Matrix with $\theta=135$

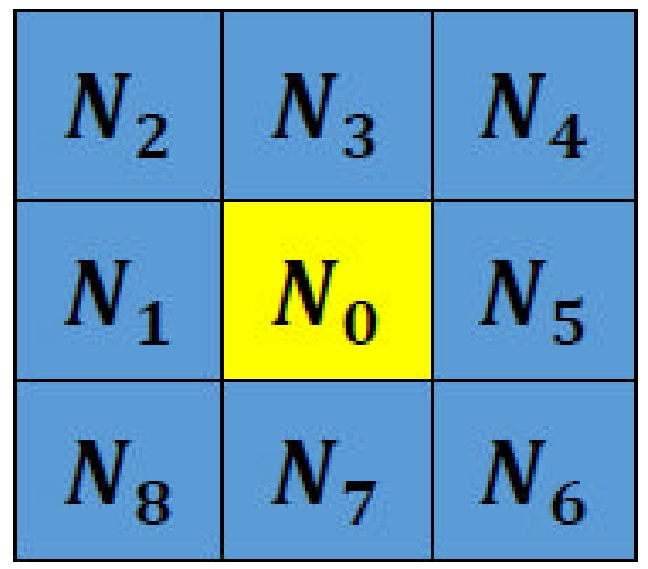

Fig. 2: The 8-neighbours

Local binary pattern (Heikkila and Pietaikinen, 2006): LBP is special case of TS. LBP use neighbors of pixel to extract texture information. We will represent LBP with 8-neighbors to center pixel like Fig. 2: The neighbors $\mathrm{N}_{\mathrm{i}}$, where $i=1, \ldots$, is encoding to 0,1 (binary encoding) by compered it with center pixel as Eq. 2 :

$$
F_{i}=\left\{\begin{array}{cc}
1 & N_{i} \geq N_{0} \\
0 & \text { else }
\end{array}\right.
$$

Then is encoding value convert to decimal number with summation the converting values of all neighbors as Eq. 3. Where the result is new value of the center pixel. See the example in Fig. 3 :

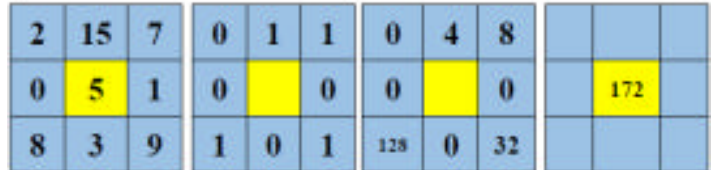

Fig. 3: Example of LBP

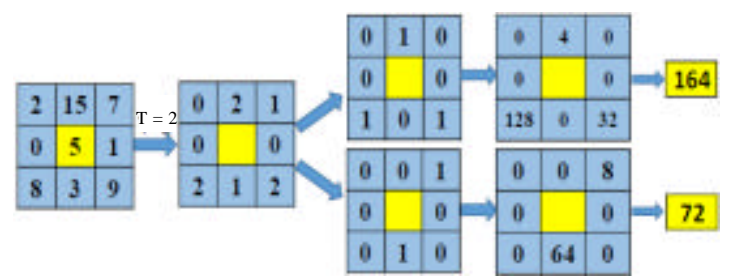

Fig. 4: Example of LTP

$$
\mathrm{LBP}_{\text {row }, \text { col }}=\sum_{\mathrm{i}=1}^{8} \mathrm{~F}_{\mathrm{i}} \times 2^{\mathrm{i}-1}
$$

Local ternary pattern (Tan and Triggs, 2007): LTP is expanded version of LBP, use the 8-neighbors of center pixel then compered each pixel with center. LTP use threshold $\mathrm{T}$ and ternary encoding as Eq. 4:

$$
F_{i}=\left\{\begin{array}{cc}
2 & \mathrm{~N}_{\mathrm{i}}-\mathrm{N}_{0}>\mathrm{T} \\
1 & \left|\mathrm{~N}_{\mathrm{i}}-\mathrm{N}_{0}\right| \leq \mathrm{T} \\
0 & \mathrm{~N}_{\mathrm{i}}-\mathrm{N}_{0}<-\mathrm{T}
\end{array}\right.
$$

Then divide into the upper and lower matrices by Eq. 5 and 6 to produce two binary matrices, as in Fig. 4. Each matrix convert to decimal number like LBP by Eq. 3 :

$$
\begin{aligned}
\text { UPPER } & =\left\{\begin{array}{cc}
1 & \text { if } \mathrm{F}_{\mathrm{i}}=1 \\
0 & \text { else }
\end{array}\right. \\
\text { LOWER } & = \begin{cases}1 & \text { if } \mathrm{F}_{\mathrm{i}}=-1 \\
0 & \text { else }\end{cases}
\end{aligned}
$$

\section{MATERIALS AND METHODS}

\section{Local Quadrant Pattern with Co-occurrence Matrix (LQP-CM)}

Local quadrant pattern: This study represent a new method called Local Quadrant Pattern with Co-occurrence Matrix (LQP-CM). This method converts each pixel to texture number that depend on the 8-neighbors around the pixel. The idea of LTP has used in this research and it summered as following: (Fig. 5 shows the framework of LQP-CM). First, calculate the difference between the center pixel $\left(\mathrm{N}_{\mathrm{c}}\right)$ with each neighbor pixels $\left(\mathrm{N}_{\mathrm{i}}\right)$ as following Eq. 7 : 


$$
\mathrm{DIF}=\mathrm{N}_{\mathrm{i}}-\mathrm{N}_{\mathrm{c}} \quad \mathrm{i}=1,2, \ldots, \mathrm{z}
$$

where, $i$ is number of neighbor pixel. The sequence of neighbors like Fig. 6. After that each opposite pixel will be in one vector to be as line Fig. 7, for example, $N_{1}$ with $\mathrm{N}_{5}$ in one line. Next, mapping this vectors to four different types of gray-level variation that shown in Fig. 8. This mapping is depending on threshold ( $\varepsilon$ ). The first type is means that the vector is very close within $\varepsilon$. Second type means that one pair of vector within $\varepsilon$ while the other exceeded. Third type describes case that vector is decreasing or increasing continuously with gray-level differences larger than $\varepsilon$. While the last type means that vector is first decreasing than increasing or the opposite (Horng, 2003):

$$
\mathrm{T}_{\mathrm{i}}\left\{\begin{array}{cc}
-2 & \left|\mathrm{p}_{1 \mathrm{i}}\right| \leq \varepsilon \cap\left|\mathrm{p}_{2 \mathrm{i}}\right| \leq \varepsilon \\
-1 & \left(\left|\mathrm{p}_{\mathrm{li}}\right| \leq \varepsilon \cap\left|\mathrm{p}_{2 \mathrm{i}}\right| \geq \varepsilon\right) \cup\left(\left|\mathrm{p}_{\mathrm{li}}\right| \geq \varepsilon \cap\left|\mathrm{p}_{2 \mathrm{i}}\right| \leq \varepsilon\right) \\
1 & \left(\mathrm{p}_{\mathrm{li}}>\varepsilon \cap \mathrm{p}_{2 \mathrm{i}}>\varepsilon\right) \cup\left(-\mathrm{p}_{\mathrm{li}}>\varepsilon \cap \mathrm{p}_{2 \mathrm{i}} \leq \varepsilon\right) \\
2 & \left(\mathrm{p}_{\mathrm{li}}>\varepsilon \cap-\mathrm{p}_{2 \mathrm{i}}>\varepsilon\right) \cup\left(-\mathrm{p}_{1 \mathrm{i}}>\varepsilon \cap \mathrm{p}_{2 \mathrm{i}} \leq \varepsilon\right)
\end{array}\right.
$$

where, $T_{i}$ is type each line, $i=1,2, \ldots, 4$. After getting four values, now produce two ternary matrices, similarly to producing LBP from LTP as next Eq. 9 and 10:

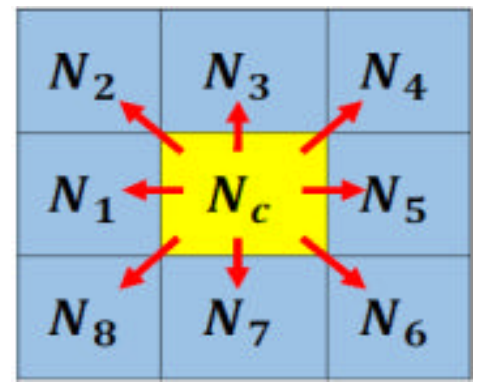

Fig. 5: The sequence of neighbors

$$
\begin{aligned}
& \mathrm{F}_{\text {upper }}=\left\{\begin{array}{cc}
1 & \text { if } \mathrm{T}_{\mathrm{i}}=1 \\
2 & \text { if } \mathrm{T}_{\mathrm{i}}=2 \\
0 & \text { else }
\end{array}\right. \\
& \mathrm{F}_{\text {lower }}=\left\{\begin{array}{cc}
1 & \text { if } \mathrm{T}_{\mathrm{i}}=1 \\
2 & \text { if } \mathrm{T}_{\mathrm{i}}=2 \\
0 & \text { else }
\end{array}\right.
\end{aligned}
$$

Finally convert this values to decimal number and summation to get new value of center pixel LQP as by Gupta et al. (2010) by Eq. 11:

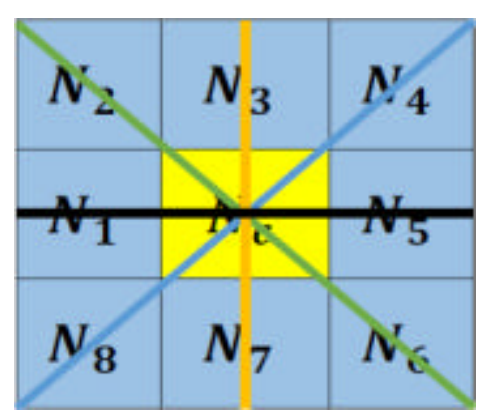

Fig. 6: Each opposite pixel in one line

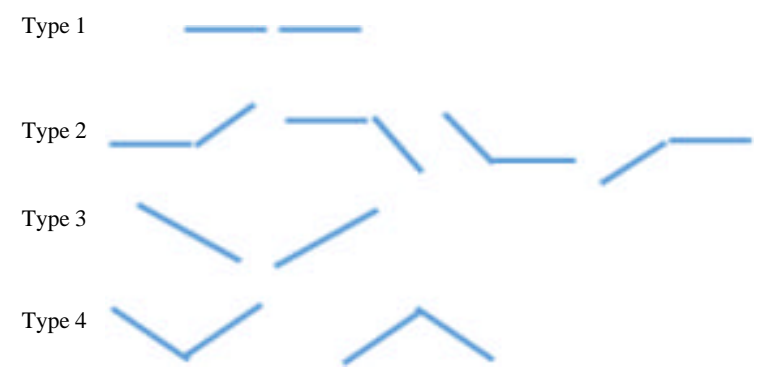

Fig. 7: Types of grey-level graphical structure variations

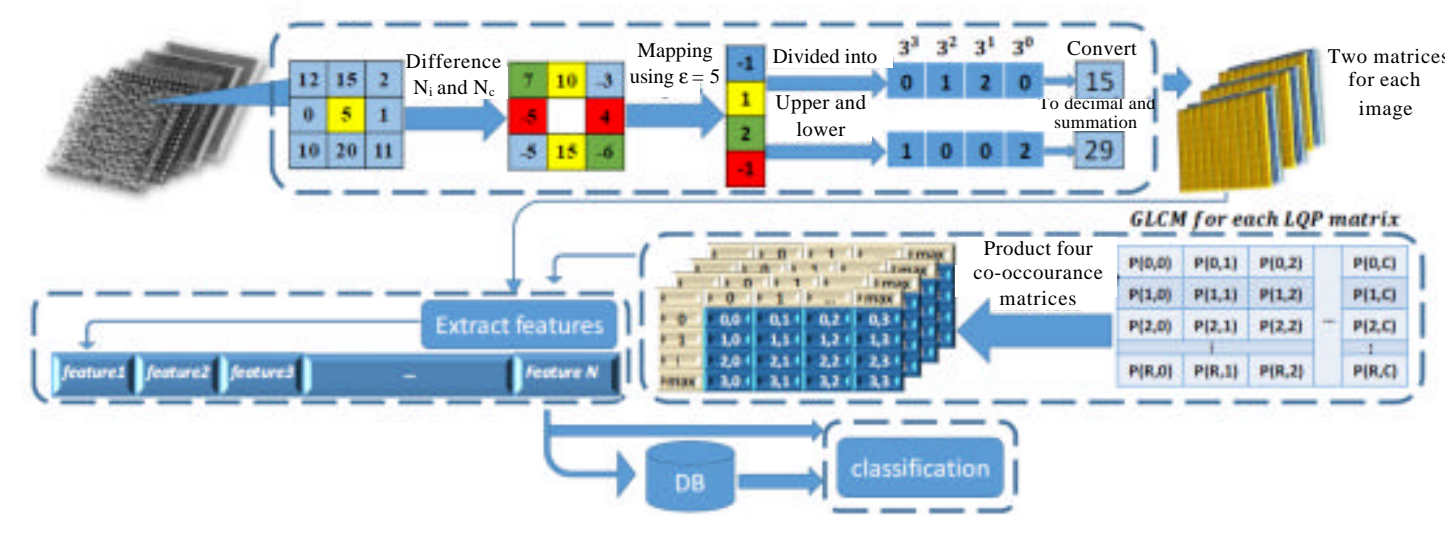

Fig. 8: Framework of LQP-CM 


$$
\mathrm{LQP}_{\text {row or col }}=\sum_{\mathrm{i}=1}^{4} \mathrm{~F}_{\text {(upper or lower), }} \times 3^{\mathrm{i}-1}
$$

Co-occurrence matrix: There are many useful features can be extracted from co-occurrence matrices. So, to extract this features, each one of LQP matrices will be the input in GLCM algorithm and the output is four matrices for each LQP upper and lower, i.e., eight matrices for original image.

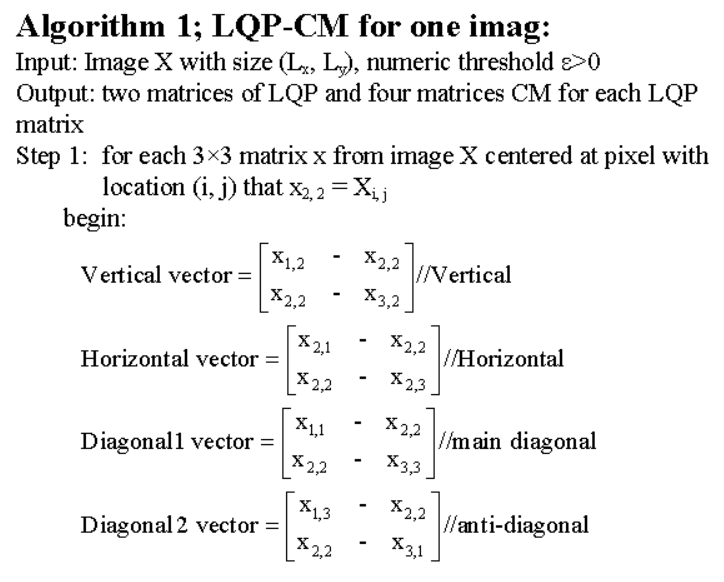

//now we see which type of gray-level belong to (the types in Fig. (7))

for $\mathrm{P}$ in \{Vertical, Horizontal, Diagonal 1, Diagonal 2\} do

if $\left|p_{1}\right| \leq \varepsilon n\left|p_{2}\right| \leq \varepsilon$ then $T(P)--2$

else if $\left(\left|p_{1}\right| \leq \varepsilon \cap\left|p_{2}\right| \geq \varepsilon\right) \cup\left(p_{1}|\geq \varepsilon \cap| p_{2} \mid \leq\right.$

ह) then $T(P)--1$

else if $\left(p_{1}>\varepsilon \cap p_{2}>\varepsilon\right) \cup\left(-p_{1}>\varepsilon \cap-p_{2}\right.$ $\leq \varepsilon)$ then $T(P)--3$

else if $\left(p_{1}>\varepsilon \cap-p_{2}>\varepsilon\right) \cup\left(-p_{1}>\varepsilon \cap p_{2} \leq\right.$

End ع) then $T(P)-2$

UPPER - T (with convert all-1 and -2 values to 0 )

Lower

$-\mathrm{T}$ (convert $(1,2$ values to 0$)$ and $(-1,-2$ values to 1,2$))$

UPPER_LQP (i, j)-sum (convert to decimal (UPPER))

LOWER_LQP (i, j)-sum (convert to decimal (UPPER))

End

step2: for each LQP matrix do:

Create four matrices with size $\left(\max \left(\mathrm{LQP}_{\mathrm{i}}\right)\right.$, $\left.\max (\mathrm{LQP})\right)$

For $\mathrm{i}=1$ to $\max \left(\mathrm{LQP}_{\mathrm{i}}\right)$

For $\mathrm{j}=1$ to $\max \left(\mathrm{LQP}_{\mathrm{i}}\right)$

Matrix $(\theta)=$ count (i have neighbor $j$ at $\theta$ and $-\theta$ direction in $X$ with distance d)

End End

Features extraction: Once LQP-CM matrices has produced, the features can extract from these matrices. There are six features has been extract, four of these features extracted from each one of LQP matrices and the other two from each CM matrix. These features are: Mean convergence, variance, homogeneity, code variance, code entropy, code similarit (Haralick et al., 1973; Horng, 2003; Marques, 2011).

Mean convergence:

$$
\mathrm{MC}=\sum_{i=0}^{\mathrm{G}-1} \frac{|\mathrm{n} \cdot \mathrm{p}(\mathrm{n}) \mu|}{\sigma}
$$

Variance:

$$
\operatorname{Var}=\sum_{\mathrm{i}=0}^{\mathrm{G}-1} \sum_{\mathrm{j}=0}^{\mathrm{G}-1}(\mathrm{p}==\max )
$$

Homogeneity:

$$
\mathrm{H}=\sum_{\mathrm{i}=0}^{\mathrm{G}-1} \sum_{\mathrm{j}=0}^{\mathrm{G}-1}(\mathrm{p}==\mathrm{min})
$$

Code variance:

$$
\mathrm{CV}=\sum_{\mathrm{i}=0}^{\mathrm{G}-1}(\mathrm{n}-\mu)^{\wedge} 2 \cdot \mathrm{p}(\mathrm{n})
$$

Code entropy:

$$
\text { C.E. }=\sum_{i=0}^{\mathrm{G}-1} \sum_{j=0}^{\mathrm{G}-1} p(i, j) \log p(i, j)
$$

Uniformity:

$$
\text { Uniformity }=\sum_{i=0}^{\mathrm{G}-1} \sum_{\mathrm{j}=0}^{\mathrm{G}-1}(\mathrm{p}(\mathrm{i}, \mathrm{j}))^{2}
$$

\section{RESULTS AND DISCUSSION}

This study analyzing the performance of LQP-CM for texture images. The performance method done by the percentage correct classification by next Eq. 18:

$$
\text { Percentage }=\frac{\text { Number of } \text { correct classification }}{\text { Total number of testing images }}
$$

Dataset: Dataset that used is Brodatz, its content 112 images where each image is one class this means its content 112 class. This study use 30 class from this dataset shown in Fig. 9. Each image rotated with sixteen different angles to create sixteen different image per class. This angles begins with $\left(0^{\circ}\right)-\left(337.5^{\circ}\right)$ with increasing (22.5). Each image with resolution $512 \times 512$ pixels and gray level. The threshold that used in LQP ( $\varepsilon$ ) will be the average of image divided by 2 .

This data dividing to two groups, training data and testing data. The experiment done by taken 3-6 images per class to training and the remainder to testing.

Classification and result: For classification, using Euclidean distance (Eq. 18) (Marques, 2011; Hussain, 2010; Shih, 2010) that calculate the distance between features of testing image and features of all training images, then take the class of minimum distance as following. 
J. Eng. Applied Sci., 14 (7): 2171-2176, 2019
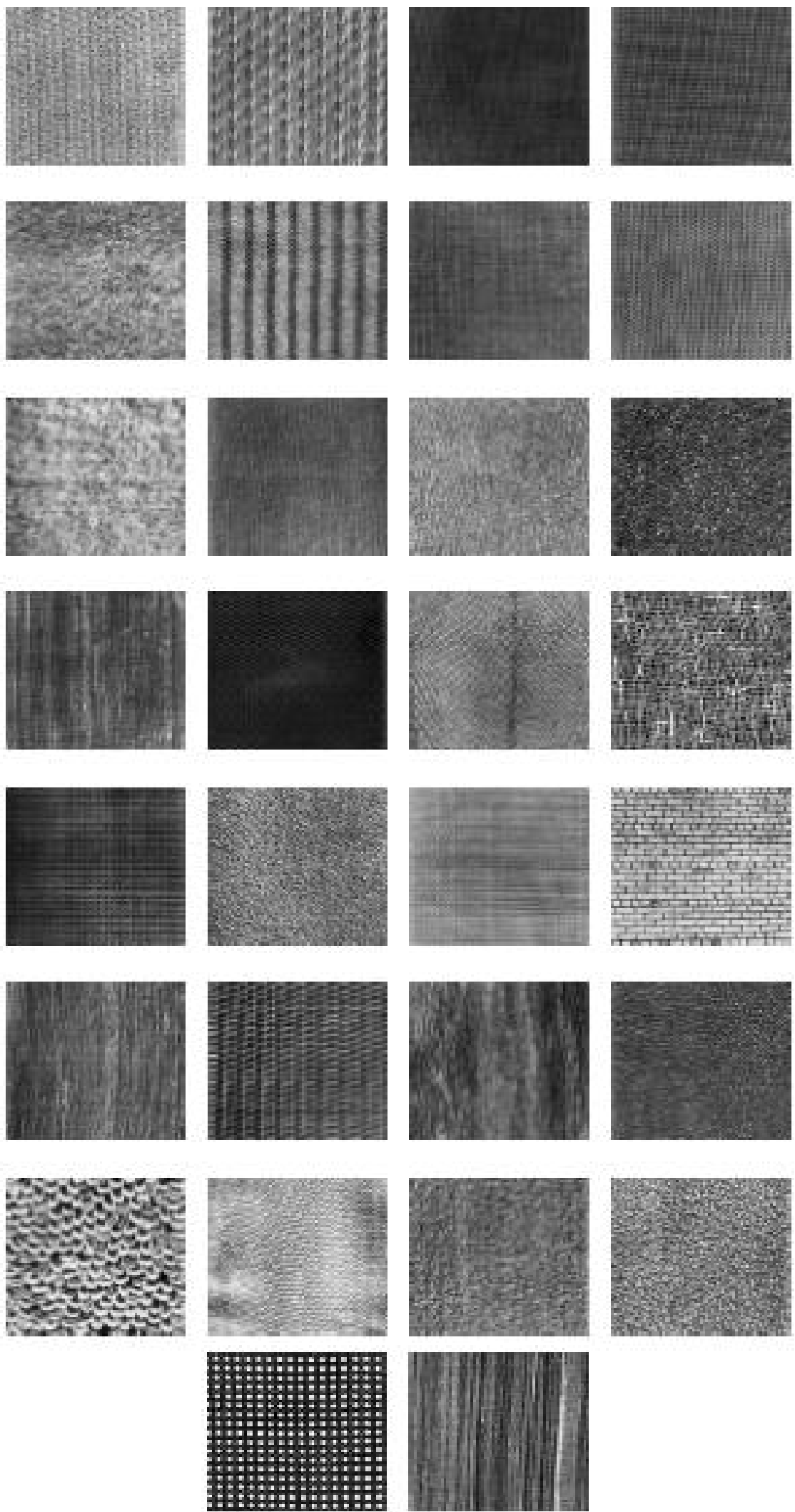

Fig. 9: 30 class from Brodatz 
Table 1: The percentage of correct classification to three methods (LQP-CM,

\begin{tabular}{|c|c|c|c|c|}
\hline Methods & 3 & 4 & 5 & 6 \\
\hline LBP-CM & - & 68.20 & 75.55 & 88.00 \\
\hline LTP-CM & 93.58 & 99.16 & 99.16 & 99.00 \\
\hline LQP-CM & 95.89 & 99.72 & 99.69 & 99.66 \\
\hline
\end{tabular}

Step 1: For each training image do:

$$
\text { Dis tan ce(i) }=\sum_{j=1}^{\mathrm{N}} \text { featur }_{\text {Taining(i) }} \text {-feature }{ }_{\text {Tsting(j) }}
$$

Step 2: MINDISTANCE $=\min ($ Distance $)$.

Step 3: Get the class of training image having minimum distance with tasting image.

The percentage of correct classification showing in Table 1. Its Compares with similar experiments using LBP and LTP with co-occurrence. Where the percentage of LQP-CM is better than others methods. If four images taken from each class (i.e., $25 \%$ ) from data to training, the result shows that correct classification for LQP-CM is $99.72 \%$, whilst LBP-CM is $75.55 \%$ and LTP-CM is $99.16 \%$ (Fig. 9).

\section{CONCLUSION}

In this study, local quadrant pattern with co-occurrence matrix is proposed. LQP-CM is new version of local patterns where it uses quadrant encoding. The proposed method, that used to extract features from texture images for classification is found to be better when compared with LBP and LTP. The result shows that LQP-CM gives high accuracy even when the training data is few.

\section{REFERENCES}

Gupta, R., H. Patil and A. Mittal, 2010. Robust order-based methods for feature description. Proceedings of the IEEE International Conference on Computer Vision and Pattern Recognition (CVPR), June 13-18, 2010 , IEEE, San Francisco, California, USA., ISBN:978-1 4244-6984-0, pp: 334-341.

Haralick, R.M., 1979. Statistical and structural approaches to texture. Proc. IEEE, 67: 786-804.

Haralick, R.M., K. Shanmugam and I.H. Dinstein, 1973. Textural features for image classification. IEEE Trans. Syst. Man Cybern., SMC-3: 610-621.

He, D.C. and L. Wang, 1991. Texture features based on texture spectrum. Pattern Recogn., 24: 391-399.

Heikkila, M. and M. Pietaikinen, 2006. A texture-based method for modeling the background and detecting moving objects. IEEE Trans. Pattern Anal. Mach. Intell., 28: 657-662.

Horng, M.H., 2003. Texture feature coding method for texture classification. Opt. Eng., 42: 228-283.

Hussain, Z.M., 2010. An Introduction to Digital Image Processing. University of Kufa Press, Kufa, Najaf, Iraq,

Marques, O., 2011. Practical Image and Video Processing Using MATLAB. 1st Edn., Wiley-IEEE Press, USA., ISBN 13: 9780470048153, Pages: 696.

Shih, F.Y., 2010. Image Processing and Pattern Recognition: Fundamentals and Techniques. John Wiley \& Sons, Hoboken, New Jersey, USA., ISBN:9780470590409, Pages: 552.

Tan, X. and B. Triggs, 2007. Enhanced local texture feature sets for face recognition under difficult lighting conditions. Proceedings of the International Workshop on Analysis and Modeling of Faces and Gestures, October 20, 2007, Springer, Berlin, Germany, ISBN:978-3-540-75689-7, pp: 168-182. 